\title{
Relationship between Working Clothes and Oxidative Stress in Agricultural Workers
}

\author{
Yim DH, Eom SY, Kim YD* and Kim H \\ Department of Preventive Medicine, Chungbuk National University, Cheongju, Korea
}

*Corresponding author: Yong-Dae Kim Department of Preventive Medicine, College of Medicine Chungbuk, Cheongju, Korea, Tel: +82-43-261-2845; Email: ydkim@cbu.ac.kr

\section{Short Communication}

Volume 3 Issue 3

Received Date: June 08, 2018

Published Date: June 15, 2018

\section{Abstract}

Agricultural workers are frequently exposed to ultraviolet (UV) radiation, which causes various diseases by inducing oxidative stress. This study aimed to assess the effects of working clothes on oxidative stress in the body. A total of 101 male and female agricultural workers were enrolled in this study. We measured urinary levels of total antioxidant capacity (TAC), an antioxidant indicator, and thiobarbituric acid reactive substance (TBARS), an oxidative stress indicator. The urinary TAC of individuals who wore long pants and long-sleeved tops while working was significantly higher than that of individuals who wore short pants and short-sleeved tops. Urinary TBARS concentrations were not statistically significant but showed a tendency to decrease proportionally when wearing clothing that covered more exposed skin. We showed that shielding from UV radiation using clothing increased antioxidant capacity and also suppressed oxidative stress generation. The result of this pilot study suggests the need for further studies to confirm these relationships.

Keywords: Agricultural workers; UV exposure; Working clothes; Oxidative stress

Abbreviations: TAC: Total Antioxidant Capacity; UV: Ultraviolet; TBARS: Thiobarbituric Acid Reactive Substance; ANCOVA: Analysis Of Covariance (ANCOVA).

\section{Introduction}

Exposure to ultraviolet (UV) radiation is known to cause eye diseases such as cataracts, macular degeneration, and pterygium. UV exposure is also closely related to the development of various skin diseases, including skin cancer $[1,2]$. Sunlight contains a large proportion of UV-A rays, most of which are absorbed by the epidermis; however, about $10 \%$ of UV-A radiation can penetrate the subcutaneous layer [3]. Because UV-A rays have less energy than UV-B or UV-C, they cause indirect adverse effects in humans via generation of oxidative stress $[4,5]$.

It is known that farmers are highly exposed to sunlight and have a higher incidence of ocular diseases or skin cancer than the general population owing to UV exposure [6,7]. Therefore, prevention of UV exposure among farmers is very important for maintaining health. However, there have been few studies evaluating the effects of oxidative stress and the protective effect of the work clothes used by farmers. The most fundamental way 
to block UV exposure is to cover as large an area of the skin as possible so that it is not exposed directly to UV light. Several studies have shown that the use of sunscreen reduces UV exposure and reduces oxidative stress $[1,8]$. However, considering that most farmers in Korea are older, it is difficult for them to use sunscreens effectively. Therefore, it may more effective for older farmers to use clothing, such as a shirt or a hat, rather than use expensive sunscreens to prevent UV exposure. Long-sleeved shirts or long pants can be expected to result in lower exposure to UV light than short sleeves or short pants; however, there are few studies that have evaluated this. The present work is a preliminary study to confirm this hypothesis by investigating the relationship between the types of clothing used by agricultural workers during work and levels of oxidative stress, total antioxidant capacity (TAC), and thiobarbituric acid reactive substance (TBARS).

\section{Participants and Methods}

This study enrolled 101 healthy volunteer agricultural workers, both men and women, who mainly worked in the field Orin a greenhouse in the Chungbuk area of Korea. After thoroughly explaining the study, written informed consent was obtained from participants. We used a questionnaire to collect demographic information and data about smoking habits and agricultural work. Spot urine was collected from participants every morning during the study (5 days) and stored at $-20^{\circ} \mathrm{C}$ until testing. Urinary TAC and lipid peroxidation levels were subsequently measured. This study received approval from the Chungbuk University Institutional Review Board (CBNU-IRB-2011-BQ02). Study participants were divided into three groups according to the types of work clothing used, as follows; group 1 wore a short-sleeved top and short pants, group 2 wore a short-sleeved top and long pants or a long-sleeved top and short pants, and group 3 wore a long-sleeved top and long pants.

Urinary TAC was evaluated by copper (II) reduction assay using bath ocuproine disulfonic acid disodium salt (CUPRAC-BCS) for total antioxidant capacity assessment reported by Apak, et al. with slight modification [9]. Briefly, each urine specimen was diluted 1:40 with 0.25 mM BCS in phosphate buffer (pH 7.4) and $200 \mu \mathrm{L}$ of each sample was placed in each well of a 96-well plate. Then, the basal absorbance was measured at $490 \mathrm{~nm}$ by microplate reader (Bio-Rad, Hercules, CA, USA). After adding $50 \mu \mathrm{L}$ of $0.5 \mathrm{mM} \mathrm{CuSO}_{4}$ solution, the reaction mixture was incubated for $3 \mathrm{~min}$ at room temperature, and then the reaction was stopped with $50 \mu \mathrm{L}$ of $0.01 \mathrm{M}$ EDTA. Finally, the absorbance was again measured at 490 $\mathrm{nm}$ and the difference in the absorbance before and after the reaction was used as the TAC level.

To determine urinary lipid peroxidation, standard solutions were prepared by dissolving the required amounts of 1, 1, 3, 3-tetramethoxypropane in 40\% ethanol to yield $2,2.5,5$, and $10 \mu \mathrm{M}$ solutions. A total $50 \mu$ Leach of urine sample and the standard solutions was mixed with $50 \mu \mathrm{L}$ of $0.05 \%$ butylated hydroxytoluene, 150 $\mu \mathrm{L}$ of $0.1125 \mathrm{~N} \mathrm{HNO}_{3}$, and $150 \mu \mathrm{L}$ of $42 \mathrm{mM}$ TBA. After a 1hr incubation at $95^{\circ} \mathrm{C}$, the mixture was cooled on ice for 5 $\min$. Then, $300 \mu \mathrm{L}$ of $n$-butanol was added to the cooled mixture, and the solution was centrifuged for $5 \mathrm{~min}$ at $1,500 \mathrm{rpm}$ and $4^{\circ} \mathrm{C}$. The supernatant was used for analyses. Using an auto sampler (SIL-10ADvp; Shimadzu, Japan), $10 \mu$ Lof each supernatant was injected into an HPLC system fitted with a fluorescence detector (RF10AxL; Shimadzu) and a reverse-phase column (TSK-gel; TOSOH, Japan). The fluorescence wavelengths were set at $\lambda \mathrm{ex}=515 \mathrm{~nm}$ and $\lambda \mathrm{em}=553 \mathrm{~nm}$. The mobile phase consisted of a mixture of $50 \mathrm{mM} \mathrm{KH} 2 \mathrm{PO} 4(\mathrm{pH}$ 6.8), methanol, and acetonitrile $(65: 20: 15, \mathrm{v} / \mathrm{v} / \mathrm{v})$, and the flow rate was $1.0 \mathrm{~mL} / \mathrm{min}$. The external standard method was applied to the TBARS quantification methods. The urinary creatinine concentration was measured using the Jaffe method, and urinary TAC and TBARS levels were adjusted according to creatinine concentration. Statistical analysis was performed using IBM SPSS version 24.0 (IBM Corp., Armonk, NY, USA). Analysis of covariance (ANCOVA) and analysis of covariance (ANOVA) with post hoc analysis were used to test differences between the groups for the various parameters. Statistical significance was set at $\mathrm{p}<0.05$.

\section{Results and Discussion}

Participants in group 1 were relatively younger (age $63.12 \pm 16.08$ years) than those in the other groups; however, the mean age of participants was not significantly different among the three groups, according to the type of clothing worn during agricultural work. The mean daily exposure time of participants in group 3 was significantly longer than that of group $1(p=0.032)$, suggesting that individuals who work long daylight hours tend to wear clothing that covers the skin relatively more completely (long-sleeved tops and long pants). Group 2 included the most men (66.7\%) whereas group 3had the most women (70.8\%).Agricultural work was classified into two types: field work and greenhouse work. There was no significant difference in the type of work clothes preferred according to work type or smoking status (Table 1). 


\section{Advances in Clinical Toxicology}

\begin{tabular}{|c|c|c|c|c|}
\hline & Group1a $^{a}$ & Group 2 & Group 3 & p-value ${ }^{b}$ \\
\hline $\mathrm{N}$ & 8 & 38 & 55 & \\
\hline Age, yr & $63.12 \pm 16.08$ & $69.63 \pm 9.18$ & $67.46 \pm 10.94$ & 0.272 \\
\hline Work time under daylight/day (hrs) & $2.00 \pm 1.31$ & $2.56 \pm 1.20$ & $3.09 \pm 1.36$ & $0.032^{\mathrm{c}}$ \\
\hline \multicolumn{5}{|l|}{ Sex, n (\%) } \\
\hline Male & $3(37.5)$ & $24(63.2)$ & $9(16.4)$ & $<0.001$ \\
\hline Female & $5(62.5)$ & $14(36.8)$ & $46(83.6)$ & \\
\hline Work type, n (\%) & & & & 0.429 \\
\hline Field & $7(87.5)$ & $26(68.4)$ & $40(72.7)$ & \\
\hline Greenhouse & $1(12.5)$ & $12(31.6)$ & $15(17.3)$ & \\
\hline Smoking status, n (\%) & & & & 0.282 \\
\hline Smoker & $8(100)$ & $33(86.8)$ & $53(96.4)$ & \\
\hline Nonsmoker & $0(0.00)$ & $5(13.2)$ & $2(3.6)$ & \\
\hline
\end{tabular}

Table 1: Distribution of demographic factors in study participants.

aGroup1: short-sleeved shirts and short pants.

Group 2: short pants and long-sleeved shirts or vice versa.

Group 3: long-sleeved shirts and long pants.

bp-value by ANOVA with post hoc analysis or chi-square test.

cSignificantly different by Duncan test: Group 1 ₹ Group 3.

We compared urinary TAC and TBARS concentrations by group, with adjustment for variables that were statistically significant in the univariate analysis and that are known to have an important role in the development of oxidative stress. As a result, urinary TAC was $0.33 \pm 0.04,0.44 \pm 0.02$, and $0.45 \pm 0.02 \mathrm{mMUA}$ equiv $/ \mathrm{mM}$ creatinine in groups 1,2 , and 3 , respectively. The results of posthoc analysis showed that there was a statistical significance between groups 1 and 2, and between groups 1 and 3. These results indicate that wearing clothing that covers more of the skin (long-sleeved tops and long pants) during agricultural work greatly suppresses oxidative stress due to UV exposure. In addition, urinary TBARS concentrations were $3.20 \pm 0.50,2.40 \pm 0.26$, and
$2.10 \pm 0.21 \mu \mathrm{mol} /$ Mcreatinine in groups 1,2 , and 3, respectively (Figure 1). TBARS showed a dose-dependent decrease according to use of working clothes with a greater degree of skin protection, even though this was not statistically significant, which may due to the small sample size. These results suggest that the clothing used during agricultural work may affect not only the generation of oxidative stress but also antioxidant ability. Therefore, based on the results of this preliminary study, it is necessary to evaluate how various oxidative stress indicators, including reactive oxygen species and the expression of various antioxidant enzymes, vary depending on the type of work clothes used during agricultural work.
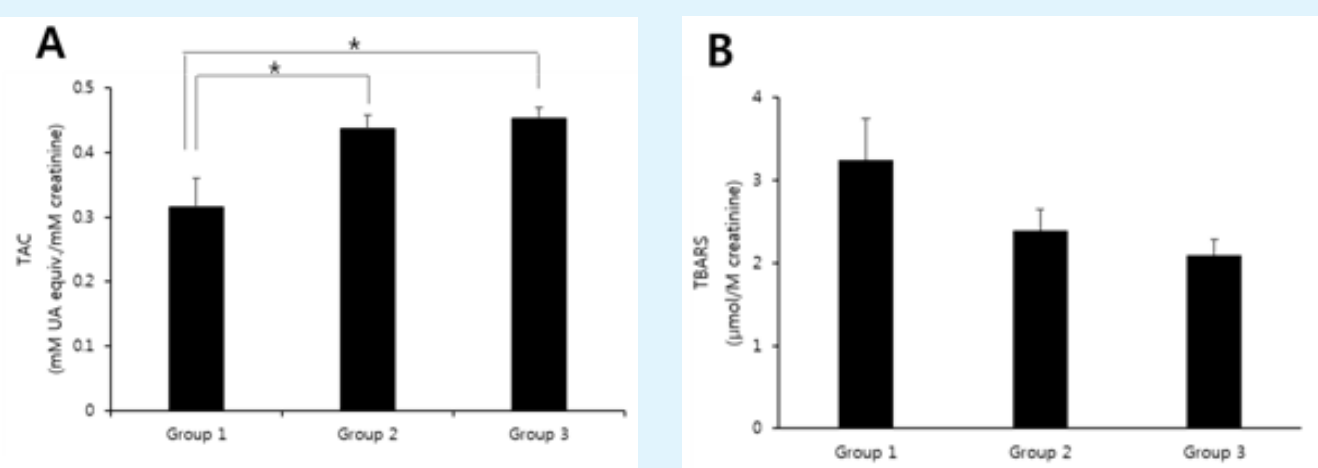

Figure 1: Comparisons of urinary TAC (A) and TBARS (B) levels according to group. The graph represents mean \pm SEM with adjustment for age, sex, type of work, smoking status, and working time under the daylight /day. *shows statistical significance with ANCOVA with Bonferroni correction. 


\section{Advances in Clinical Toxicology}

Reactive oxygen species generated by UV rays can cause DNA damage, such as DNA single- and doublestrand breaks or base substitution. Therefore, people who are exposed to UV rays should wear appropriate protective clothing to block sunlight, to prevent the development of skin cancer $[10,11]$.However, agricultural workers are one of the representative groups that are occupationally exposed to sunlight. There has been a lack of effort to prevent various diseases caused by UV radiation exposure in Korea overall. One of the main reasons for this is that the harmful effects of UV exposure are unclear, unlike those owing to pesticides. In particular, older farmers tend to perceive that UV exposure is not a serious health hazard. Therefore, it is necessary to continuously educate agricultural workers and publicize the harm caused to the human body by UV radiation exposure, to increase public interest in using caution when exposed to UV light.

Several previous studies have reported that sunscreens have a protective effect against oxidative stress caused by UV exposure [6,8]. However, considering that most farmers in Korea are older adults, it is not easy for them to use sunscreens several times a day, every day. By comparison, UV protection from clothing is a very effective method in terms of cost and simplicity for older farmers.

There are some limitations in this study with respect to the control of other factors including diet, which may affect oxidative stress. However, considering that all participants were agricultural workers living in the same area and were of similar age, it was thought that eating habits among participants would not show a large difference. Additionally, we used the average value of urine samples for 5 consecutive days, to minimize daily variations. The possibility of selection bias cannot be excluded due to the lack of random allocation, and the present study has lower statistical power because of the small sample size. There is also a possibility that UV ray transmittance may differ depending on the type of material of the clothing worn. Although this study has several limitations, we showed sufficient probability that work clothes may suppress oxidative stress during agricultural work, as a preliminary result. This study is important in that it is the first to grasp the relationship between the type of clothing used during agricultural work and the occurrence of oxidative stress in the body among older farmers in Korea. It is necessary to confirm the results in a well-designed, large-scale epidemiological study in the future.

\section{Acknowledgements}

This work was supported by the intramural research grant of Chungbuk National University in 2015.

\section{References}

1. Kaur A, Thatai P, Sapra B (2014) Need of UV protection and evaluation of efficacy of sunscreens J Cosmet Sci 65(5): 315-345.

2. Mancebo SE, Wang SQ (2014) Skin cancer: role of ultraviolet radiation in carcinogenesis. Rev Environ Health 29(3): 265-273.

3. The International Agency for Research on Cancer (1992) IARC monographs on the evaluation of carcinogenic risks to humans. Solar and ultraviolet radiation. IARC Monogr Eval Carcinog Risks Hum 55: 1-316.

4. Altieri F, Grillo C, Maceroni M, Chichiarelli S (2008) DNA damage and repair: from molecular mechanisms to health implications. Antioxid Redox Signal 10(5): 891-937.

5. Svobodová AR, Galandáková A, Sianská J, Doležal D, Ulrichová J, et al. (2011) Acute exposure to solar simulated ultraviolet radiation affects oxidative stress-related biomarkers in skin, liver and blood of hairless mice. Biol Pharm Bull 34(4): 471-479.

6. Silk KJ, Parrott RL (2006) All or nothing or just a hat? Farmers' sun protection behaviors. Health Promot Pract 7(2): 180-185.

7. Fragar L, Depczynski J, Lower T (2011) Mortality patterns of Australian male farmers and farm anagers. Aust J Rural Health 19(4): 179-184.

8. Kim YD, Yim DH, Eom SY, Lee JY, Kim H (2017) The effect of sunblock against oxidative stress in farmers: a pilot study. J Biomed Res 31(4): 344-349.

9. Apak R, Güçlü K, Demirata B, Ozyürek M, Celik SE, et al. (2007) Comparative evaluation of various total antioxidant capacity assays applied to phenolic compounds with the CUPRAC assay. Molecules 12(7): 1496-1547.

10. Kato M, Iida M, Goto $Y$, Kondo T, Yajima I (2011) Sunlight exposure-mediated DNA damage in young adults. Cancer Epidemiol Biomarkers Prev 20(8): 1622-1628. 


\section{Advances in Clinical Toxicology}

11. Kil HN, Eom SY, Park JD, Kawamoto T, Kim YD, et al. (2014) A rapid method for estimating the levels of urinary thiobarbituric Acid reactive substances for environmental epidemiologic survey. Toxicol Res 30(1): 7-11. 